\title{
Caracterización de la
}

autogestión del aprendizaje en los estudiantes universitarios de derecho y la licenciatura en Filosofía y Lengua Castellana en la Universidad Santo Tomás (Colombia)"

\author{
Francisco Alonso Chica Cañas"* \\ InMACULADa AZnar Díaz ${ }^{* * *}$
}

* Proyecto finalizado. Financiado por la Universidad Santo Tomás.

** Licenciado en Educación Filosofía y Letras. Universidad Santo Tomás; Magister en Educación Filosofía Latinoamericana. Universidad Santo Tomás; Magister en Docencia Universitaria. Universidad de la Salle; Especialista en Pedagogía para el Desarrollo del Aprendizaje Autónomo. UNAD; Especialista en Gerencia de Instituciones de Educación Superior. Universidad Santo Tomás; Doctor en Currículum, Profesorado e Instituciones Educativas de la Universidad de Granada. España. Del mismo modo, ejerció como Decano de la Facultad de Educación durante seis años en la Universidad Santo Tomás. Actualmente forma parte de los grupos de Investigación Fray Saturnino Gutiérrez, O. P. Filosofía de la Educación en Colombia y América Latina. Universidad Santo Tomás. Correo electrónico: autonomiafra9@gmail.com

*** Profesora Titular de Universidad en el Departamento de Didáctica y Organización Escolar de la Facultad de Ciencias de la Educación de la Universidad de Granada, Doctora en Ciencias de la Educación por la misma Universidad, Licenciada en Psicopedagogía y Maestra de Educación Primaria. Fue profesora durante dos cursos académicos en el Departamento de Educación de la Universidad de Córdoba. Ha ejercido como Vicedecana de Estudiantes y Extensión universitaria durante 


\section{Introducción}

En este estudio se pretende caracterización de la autogestión del aprendizaje de los estudiantes universitarios del programa de Derecho y la licenciatura en Filosofía y Lengua Castellana en la Universidad Santo Tomás (Colombia), teniendo en cuenta el uso que hacen del Internet en la solución de los problemas académicos, el empleo del conocimiento declarativo para acceder a un aprendizaje significativo, el dominio de habilidades de pensamiento de orden superior en torno al papel que juega la lectura en el estudio, la actitud positiva para enfrentar las situaciones negativas de la vida y las condiciones ambientales adecuadas para la concentración y la atención en el estudio.

Esto es el resultado del aporte de pedagogos y de otros pensadores de diversos ámbitos disciplinares que coinciden en el desarrollo de una autonomía intelectual, moral y científica que gestione los aprendizajes para verificar lo aprendido. Por consiguiente, no se entiende la autonomía si no existe la autogestión del aprendizaje que significa emplear de forma eficiente y eficaz las mediaciones para problematizar el conocimiento con una percepción del uso y generación de conocimiento, empleando el potencial de una inteligencia encarnada, tal como lo sostiene (Varela, 2000).

\section{Antecedentes de la investigación}

La autogestión del aprendizaje visto desde la pedagogía para el desarrollo del aprendizaje radica en el desempeño y en el logro de las metas de estudio que involucra la capacidad de arriesgarse para tomar la iniciativa para educarse a sí mismo con un alto sentido de responsabilidad, que conlleva la utilización de un repertorio de estrategias de aprendizaje y de recursos que contribuya a un estudio eficaz. De ahí

cuatro años. Autora de varios libros, capítulos y ha publicado gran cantidad de artículos en revistas científicas internacionales y nacionales. Coordinadora de diferentes proyectos de Innovación Docente en la Universidad de Granada y revisora en revistas nacionales e internacionales. Correo electrónico: iaznar@ugr.es 
que, para Aebli (1998) puede inferirse que la autogestión consiste en autorregular la solución de los problemas mediante un gran sentido de motivación para conocer el proceso ideal de aprendizaje, en saber hacer para orientar la propia formación personal mediante la comprobación por sí mismo acerca de lo que aprende y estar plenamente convencido del uso de lo que aprende y de quererlo hacer por voluntad personal.

Rué (2009) percibe que el aprendizaje en el aula de clase configura un pensamiento de interacción con un sentido crítico y de creatividad. Por esta razón, la autonomía implica que los aprendices tengan metas de aprendizaje o un propósito para el proyecto de vida personal, a partir de un pensamiento crítico o emancipador; igualmente, contar con un entorno enriquecedor para interactuar con las personas y los grupos, es decir, con un entorno dinámico estructurante que potencie el aprendizaje; del mismo modo, establecer un plan de autorregulación de la ruta que emprendió y cómo lo hace para lograr los propósitos que se impuso.

Otros teóricos como (Freire, 2009; Gardner, 1999; Gaskins y Elliot, 1999; Llinás, 2002; Mateos, 2001; Morin, 2001;) enfatizan en términos generales en un aprendizaje significativo y contextual que involucra acciones de compromiso para crecer en el estudio. Por esta razón, la autonomía concebida como ámbito de actuación que parte de las inteligencias múltiples, en cuanto que presenta diversas vías de acceso, como la narración, la metáfora, los conceptos, las analogías y las teorías entre otros. Lo que quiere decir, que existen diversos estilos de aprendizaje, de acuerdo con las inteligencias en las que presentan mayores experiencias los aprendices. Esto indica, que la autogestión cuenta con un rango de autorregulaciones cognitivas y metacognitivas que se apoyan en el lenguaje verbal, la precepción y valoración de la naturaleza, el movimiento del cuerpo, el ritmo de la música, las representaciones mentales del espacio, el conocimiento de sí mismo y la manera de relacionarse con los demás. Entonces, los teóricos del aprendizaje humano sostienen que la autorregulación del aprendizaje es beneficioso para alcanzar niveles de un aprendizaje ideales y complejos en torno a la formación del espíritu científico.

Todo esto nos revela que existen algunas investigaciones como la de Pérez (2010) que abordó "Las competencias para la autogestión del aprendizaje en las carreras de derecho y estudios socioculturales de la 
educación a distancia en la Universidad de Ciego de Ávila (Cuba)", señalando las dificultades de los aprendices para gestionar los conocimientos y desenvolverse mejor con el uso de las tecnologías, lo cual implicó una orientación guiada en la solución de los problemas de aprendizaje.

La investigación de Lorente (2004) titulada "Autogestión en educación física. Un estudio de caso en secundaria", la cual se concibe como una propuesta curricular de autogestión para desarrollar la autonomía en la que los aprendices construyen los conocimientos por curiosidad y por voluntad propia a partir de las experiencias personales que parten de la interacción entre los participantes y un contexto sociocultural que tiene como referente un estudio de caso.

De igual manera, se destaca la investigación de Fernández (2005) denominada "Modelo informático para la autogestión del aprendizaje para la universalización de la enseñanza”, que enfatiza en el uso de las tecnologías en torno a la solución de problemas de aprendizaje, basado en la interacción con las máquinas y la familiaridad con las herramientas tecnológicas y la organización de los contenidos para brindarle una retroalimentación a los aprendices, con el fin de integrarlos a diferentes proyectos de aprendizaje.

Además, se reseñan un número determinado de estudios (Chaves, 2014; Lorente y Joven, 2009; Ortega, 2007; Villardón y Yániz, 2011) que están relacionados con la autogestión del aprendizaje en los docentes y el desarrollo de competencias en torno a las Tics con base en la revisión de fuentes documentales, el diseño de Redes Inteligentes para promover el desarrollo de la autogestión del aprendizaje, el desarrollo de una propuesta de enseñanza - aprendizaje en torno a la Educación Física relacionado con la gestión de clase a cargo del alumnado, la relación de la autogestión del aprendizaje y la iniciativa personal para el desarrollo de la autonomía.

Entonces, en el ámbito educativo de la educación superior se necesita llevar a cabo una investigación sobre "La caracterización de la autogestión del aprendizaje en los estudiantes universitarios del programa de Derecho y de la licenciatura en Filosofía y Lengua Castellana en la modalidad presencial en la Universidad Santo Tomás (Colombia)”. Por esta razón, el interés por estudiar cómo los aprendices emplean el Internet para la solución de los problemas de aprendizaje, la utilización 
de un conocimiento declarativo para lograr un aprendizaje significativo, el impacto de la lectura en el desarrollo de habilidades de pensamiento de orden superior, la actitud positiva para enfrentar las dificultades de la vida y el modo como acceden a un clima ambiental adecuado para estudiar.

\section{Marco teórico y objetivos}

Para el desarrollo de la pedagogía del aprendizaje autónomo se requiere que los aprendices desarrollen habilidades para la autogestión, con el fin de evidenciar los logros en la apropiación de un aprendizaje significativo. La autogestión se entiende como la apropiación de un repertorio de estrategias didácticas que contribuyen a la problematización del conocimiento, al dominio de un pensamiento hipotético que conduce a la gestación de hábitos mentales de indagación e investigación científica, a la verificación de los aprendizajes en contexto social y cultural, el desarrollo de habilidades de pensamiento de orden superior para inferir, generalizar, conceptualizar y teorizar acorde con la formación del espíritu científico y la disposición de un clima interior y social apropiado para la concentración en el uso y generación de conocimiento.

La autogestión del aprendizaje apunta hacia la eficiencia y la eficacia para aprender a aprender, utilizando todos los medios y los recursos pedagógicos que conduzcan a un aprendizaje por descubrimiento, por comprensión, a un conocimiento declarativo y procedimental y a un aprendizaje para la vida. Por tanto, la autogestión se entiende como una actividad creadora dirigida a la acción, tendiente a conseguir las metas propuestas en el aprendizaje en medio del ensayo y el error, pero con la seguridad de acceder progresivamente a la certeza de la formación del espíritu científico y de la humanidad en medio de los grandes baches de la incertidumbre.

Por consiguiente, la autonomía se entiende como el modo de:

Procesar contenidos, aprender estrategias y habilidades de pensamiento para generar la capacidad de autogestión del aprendizaje, y conocer técnicas y estrategias de estudio y aprendizaje que 
permitan realizar eficientemente las dos anteriores. Recíprocamente, el enseñante deberá mediar la adquisición y desarrollo de las estrategias y habilidades mentales y de los procesos didácticos para facilitar tales operaciones. (Insuasty, 2001, p. 14)

La autogestión del aprendizaje tiene que ver directamente con los medios y mediaciones que emplea el aprendiz para lograr los propósitos del aprendizaje. Por tal razón, puede disponer de todos los medios que sean necesarios en el orden convencional y tecnológico. Sin embargo, también el aprendiz debe contar con un repertorio de estrategias para organizar, procesar y recuperar la información, para trabajar en equipo, para comunicarse y relacionarse en términos de reciprocidad con otras personas, con el fin de convertir los medios en mediaciones didácticas. Esto quiere decir que la autogestión se relaciona con la planificación del aprendizaje, el tratamiento de los contenidos, la recuperación de la información, la formulación de preguntas y respuestas, la evaluación y la construcción de conceptos con base en el modelaje del estilo de aprendizaje personal.

Insuasty (2002) expresa que la autogestión del aprendizaje requiere de estrategias didácticas que faciliten la generación de conocimiento y de estrategias de aprendizaje, con el ánimo de procesar la información de forma consciente, voluntaria, con apropiación de reglas, acciones y procedimientos por parte de los aprendices para alcanzar las metas propuestas en las tareas. Por otro lado, el acto de aprender persigue metas claras, reflexiona en forma deliberada y consciente de acuerdo con la intencionalidad o meta e involucra procesos implícitos y explícitos relacionados con el aprendizaje significativo.

Insuasty (2002) manifiesta que la autogestión del aprendizaje en términos de autoaprendizaje conlleva tres momentos que están relacionados con las personas, las tareas y el repertorio de estrategias de aprendizaje. Por esta razón, es importante:

En primer lugar, la selección consciente de las habilidades y estrategias necesarias para ejecutar bien una tarea, en segundo término, el dominio de mecanismos de autorregulación que permite evaluar 
el uso apropiado o no de habilidades y estrategias y, finalmente, el empleo de medidas de autocorrección o de mejoramiento (p. 232).

La autogestión del aprendizaje parte del principio de aprender haciendo para lograr una madurez intelectual, moral y científica. Esto quiere decir que el aprendiz debe desarrollar estrategias de adquisición y recuperación de la información en torno a las tareas. En cuanto a las relaciones interpersonales favorecer un aprendizaje de interacción social apoyado en la motivación, la autoestima, la atención, el desempeño y la actitud positiva y proactiva para afrontar las situaciones negativas de la vida. El disponer de un clima ambiental interior y externo que ayude a la concentración y a la problematización del aprendizaje, contando con los recursos físicos y tecnológicos indispensables para un aprendizaje significativo.

Como consecuencia de lo anteriormente enunciado, el objetivo radica en "establecer las características de la autogestión del aprendizaje en los estudiantes universitarios de los programas de Derecho y de la licenciatura en Filosofía y Lengua Castellana en la modalidad presencial en la Universidad Santo Tomás (Colombia)".

Del mismo modo se plantearon los siguientes objetivos específicos:

- Identificar el papel del Internet para solucionar los problemas académicos en el estudio.

- Calificar la utilidad de los conocimientos declarativos, de hechos y acontecimientos para el desarrollo del aprendizaje autónomo.

- Determinar el impacto de la lectura en el desarrollo de las habilidades de pensamiento de orden superior.

- Establecer el modo como el estudiante enfrenta el aprendizaje con una actitud positiva en el estudio.

- Evaluar las condiciones del clima ambiental para lograr un aprendizaje de concentración, atención y recuperación de la información. 


\section{Método}

La investigación tiene como referente los objetivos, el marco teórico y las categorías relacionadas con la autogestión del aprendizaje en los estudiantes universitarios del programa de Derecho y de la licenciatura en Filosofía y Lengua Castellana a través de una investigación descriptiva. La investigación parte de una encuesta o cuestionario que toma una muestra representativa relacionada con las variables planteadas anteriormente, que conlleva a un único registro por periodo de observación.

El muestreo está dirigido a los estudiantes del programa de Derecho y de la licenciatura en Filosofía y Lengua Castellana de la Universidad Santo Tomás (Colombia). Para la selección se contó con una muestra representativa de los todos los semestres de estudio, el género y la carrera que cursa; por otro lado, la población se escogió según la lista de estudiantes matriculados por curso.

La selección de la muestra incluye toda la población de estudiantes matriculados. De igual manera, el investigador conoce la población y puede escoger los elementos que considere pertinente para la muestra; por ende, se incluyó a todos los estudiantes de los semestres cursados para garantizar la representación de los niveles de estudio. En otros términos, se utilizó un diseño estratificado en donde el tamaño de la muestra corresponde a una población invitada de 1.330 estudiantes universitarios, representado en un 19,8\% de la población del programa de Derecho y un $42 \%$ de la licenciatura en Filosofía y Lengua Castellana.

A continuación, se expone el plan de análisis estadístico que contiene:

Caracterización de la población encuestada según el nivel de autogestión del aprendizaje.

- Análisis descriptivo de las respuestas a las preguntas B13 a B17.

- Asociación entre los atributos observados y referentes a la autonomía del aprendizaje.

- Medición del nivel de autogestión del aprendizaje.

- Análisis de perfiles. 
Los resultados de este estudio forman parte de la investigación de tesis doctoral de la Universidad de Granada (España) denominada "Análisis sobre la incidencia del aprendizaje autónomo en el desarrollo de las actividades en ambientes convencionales y virtuales en estudiantes universitarios de las universidades Santo Tomás y EAN (Colombia)". De ahí que, esta investigación retoma las variables relacionadas con la autogestión del aprendizaje en los estudiantes universitarios de Derecho y la licenciatura en Filosofía y Lengua Castellana de la Universidad Santo Tomás (Colombia).

\section{Resultados}

\section{Análisis descriptivo de las respuestas a las preguntas B13 a B17}

En este apartado se analizan los resultados obtenidos en la encuesta de las preguntas orientadas a evaluar la autogestión del aprendizaje, incluidas en la encuesta aplicada.

Consulta de Internet para solucionar problemas académicos.

Tabla 1. PB13. ¿Consulta el Internet como ayuda para

solucionar problemas académicos?

\begin{tabular}{|c|c|c|c|c|}
\hline \multicolumn{5}{|c|}{ Resultado } \\
\hline Respuesta & Frecuencia & Porcentaje & Frec. acumulada & $\%$ acumulado \\
\hline Nunca & 13 & 4.55 & 13 & 4.55 \\
\hline Algunas veces & 143 & 50 & 156 & 54.55 \\
\hline Siempre & 130 & 45.45 & 286 & 100 \\
\hline
\end{tabular}

\section{Comentarios}

El $(4,55 \%)$ de los estudiantes respondió que nunca realizan (consulta el Internet como ayuda para solucionar los problemas académicos) y la otra población de estudiantes sostuvo que algunas veces $(50 \%)$ utilizan el Internet en el estudio. En cambio, el grupo restante de estudiantes $(45.45 \%)$ afirman que siempre navegan para indagar los temas 
relacionados con las asignaturas. La autogestión en el Internet posibilita a los estudiantes de Derecho acceder a las sentencias nacionales e internacionales y a los estudiantes de la licenciatura en Filosofía y Lengua Castellana a los tratados filosóficos, lo que posibilita un desarrollo de la autonomía intelectual, moral y científica. Sin embargo, un grupo representativo de estudiantes sostienen que los aparatos tecnológicos se constituyen en distractores que inciden en el bajo rendimiento académico; aunque para otros estudiantes es una herramienta valiosa para profundizar en los contenidos de la asignatura.

Califique de 1 a 5 la necesidad de utilizar los datos, hechos y acontecimientos de las asignaturas que cursa, para formar pensamientos o conceptos con sentido de utilidad en la vida cotidiana. (5 muy importante).

Tabla 2. PB14. Calificación de la necesidad de utilizar hechos académicos para la formación

\begin{tabular}{|l|r|r|r|r|}
\hline \multicolumn{1}{|c|}{ Respuesta } & \multicolumn{1}{|c|}{ Frecuencia } & \multicolumn{1}{c|}{ Porcentaje } & Frec. acumulada & \% acumulado \\
\hline 2 & 3 & 1.05 & 3 & 1.05 \\
\hline 3 & 42 & 14.69 & 45 & 15.73 \\
\hline 4 & 118 & 41.26 & 163 & 56.99 \\
\hline $\begin{array}{l}5 \text { (muy } \\
\text { importante) }\end{array}$ & 123 & 43.01 & 286 & 100 \\
\hline
\end{tabular}

\section{Comentarios}

Los estudiantes consideran necesario y muy necesario $(84,27 \%)$ la (utilización de datos, hechos y acontecimientos de las asignaturas que cursan), para formar pensamientos o conceptos con sentido de utilidad en la vida cotidiana. Esto indica que el $84,27 \%$ de los estudiantes entienden que a través del lenguaje y los hechos cotidianos de la vida se es capaz de relacionar y transferir conceptos a la vida cotidiana para captar el sentido y el significado en contexto social, cultural o de la vida práctica como tal. El conocimiento declarativo posibilita un aprendizaje significativo porque afianza en la memoria los hechos 
y los acontecimientos, permitiendo una conceptualización que cuenta con una huella perdurable en la memoria. También se aprecia que una minoría de estudiantes $(15,74 \%)$ no consideran necesarios los conocimientos declarativos, impidiendo crear un puente entre las categorías conceptuales y el significado que proporciona el conocimiento fáctico.

La lectura de un escrito le permite: 1 clasificar la información, 2. formular conclusiones, 3. tomar decisiones, 4. comprender teorías.

Tabla 3. PB15. La lectura de un escrito le permite

(Seleccione los 3 más importantes)

\begin{tabular}{|r|r|r|r|r|}
\hline \multicolumn{5}{|c|}{ Resultado } \\
\hline Respuesta & \multicolumn{1}{|c|}{ Frecuencia } & \multicolumn{1}{c|}{ Porcentaje } & Frec. acumulada & $\%$ acumulado \\
\hline Seleccionó 1 & 59 & 20.63 & 59 & 20.63 \\
\hline Seleccionó 2 & 15 & 5.24 & 74 & 25.87 \\
\hline Seleccionó 3 & 212 & 74.13 & 286 & 100 \\
\hline
\end{tabular}

\section{Comentarios}

Los estudiantes consideran que (la lectura de un escrito le permite) tomar decisiones $(74,13 \%)$, clasificar la información $(20,63 \%)$ y formular conclusiones $(5,24 \%)$. Este dato es importante porque el estudiante se apropia de los conceptos de los libros y lo transfiere a la vida práctica o cotidiana, facilitándole verificar la teoría con la praxis, lo que contribuye al desarrollo de habilidades de pensamiento de orden superior como la clasificación, la comparación, la generalización, la categorización de conceptos y la formulación de conclusiones. La habilidad de toma de decisiones posibilita la indagación, el estudio y la adquisición del conocimiento de una manera más rápida y la formación de un pensamiento crítico para afrontar los dilemas de la futura vida profesional. 


\section{Al enfrentar su aprendizaje, ¿Usted tiene una actitud positiva?}

Tabla 4. PB16. ¿Al enfrentar su aprendizaje usted tiene una actitud positiva?

\begin{tabular}{|c|c|c|c|c|}
\hline \multicolumn{5}{|c|}{ Resultado } \\
\hline Respuesta & Frecuencia & Porcentaje & Frec. acumulada & $\%$ acumulado \\
\hline $\mathrm{Si}$ & 274 & 95.8 & 274 & 95.8 \\
\hline No & 12 & 4.2 & 286 & 100 \\
\hline
\end{tabular}

\section{Comentarios}

Los estudiantes afirman que (al enfrentarse al aprendizaje tienen una actitud positiva) $(95.8 \%$ ) y un grupo reducido de estudiantes $(4,2 \%)$ no son positivos para asumir el estudio. Esto indica que el estudiante es capaz de enfrentar las circunstancias negativas de la vida y asumir la frustración para postergar el cumplimiento de las metas personales. Esto obedece a que tiene una alta autoestima de sí mismo para salir adelante por motivación propia y el auto compromiso debido a la inversión económica realizada por los padres de familia en la matrícula. Entonces, la actitud positiva estriba en la lucha, en el esfuerzo y la perseverancia por alcanzar las metas personales, teniendo como ingrediente la motivación intrínseca.

\section{Seleccione las 3 condiciones que usted considera} más importantes para estudiar.

\section{Biblioteca, 2. Computador, 3. Escritorio. 4. Silencio para la concentración, 5. Ninguna de los anteriores.}

Tabla 5. PB17. Calificación según la selección realizada

\begin{tabular}{|c|r|r|r|r|}
\hline Calificación & \multicolumn{5}{|c|}{ Resultado } & \\
\hline 1 & 7 & 2.45 & 7 & 2.45 \\
\hline 2 & 2 & 0.7 & 9 & 3.15 \\
\hline 3 & 3 & 1.05 & 12 & 4.2 \\
\hline 4 & 4 & 1.4 & 16 & 5.59 \\
\hline 5 & 25 & 8.74 & 41 & 14.34 \\
\hline
\end{tabular}




\begin{tabular}{|c|r|r|r|r|}
\hline Calificación & \multicolumn{5}{|c|}{ Frecuencia } & \multicolumn{1}{|c|}{ Porcentaje } & Frec. acumulada & \% acumulado \\
\hline 7 & 4 & 1.4 & 45 & 15.73 \\
\hline 8 & 6 & 2.1 & 51 & 17.83 \\
\hline 9 & 39 & 13.64 & 90 & 31.47 \\
\hline 10 & 63 & 22.03 & 153 & 53.5 \\
\hline 11 & 55 & 19.23 & 208 & 72.73 \\
\hline 12 & 78 & 27.27 & 286 & 100 \\
\hline
\end{tabular}

Los valores de la siguiente tabla para la variable PB17, corresponden a:

12: Seleccionó el silencio, el escritorio y una biblioteca.

11: Seleccionó el silencio, el escritorio y un computador.

10: Seleccionó el silencio, la biblioteca y un computador.

9: Seleccionó el escritorio, un computador y una biblioteca.

\section{Comentarios}

Los estudiantes $(27,27 \%)$ manifiestan que la (condición más importante para estudiar) es el silencio, el escritorio y una biblioteca; seguido de un grupo de estudiantes $(19,23 \%)$ que seleccionó el silencio, el escritorio y un computador; además, otro grupo de estudiantes $(22,03 \%)$ considera el silencio, la biblioteca y un computador. Por tanto, se percibe que un grupo representativo de estudiantes seleccionó el escritorio, un computador y una biblioteca. Se aprecia que el $68.53 \%$ de los estudiantes perciben que el clima ambiental favorece la concentración y la atención en el estudio, apoyado en unas muy buenas relaciones interpersonales, tanto de la familia como de los compañeros. Esto se evidencia cuando se acercan los parciales y las evaluaciones; además de contar con recursos ambientales apropiados que estimulen la autogestión del aprendizaje en cuanto un clima interior y un clima social. 


\section{Asociación entre los atributos observados y referentes a la autonomía del aprendizaje}

La siguiente es la matriz de los valores de la estadística Chi cuadrada para medir la asociación entre las preguntas B13 a B17 orientadas a valorar el nivel de autogestión del aprendizaje.

\section{Resultado}

Tabla 6. Resultado

\begin{tabular}{|l|l|l|l|l|}
\hline \multicolumn{1}{|c|}{ B13 } & \multicolumn{1}{|c}{ B14 } & \multicolumn{1}{|c}{ B15 } & \multicolumn{1}{l}{ B16 } \\
\cline { 1 - 3 } B13 & NA & \multicolumn{2}{|l}{} \\
\cline { 1 - 3 } B14 & 0.6644 & NA & \multicolumn{1}{|l}{} \\
\cline { 1 - 3 } B15 & 0.4322 & 0.1364 & NA & \\
\hline B16 & 0.6761 & 0.7263 & 0.5249 & NA \\
\hline B17 & 0.7599 & 0.1520 & 0.0001 & 0.4003 \\
\hline
\end{tabular}

\section{Comentario}

La asociación entre la pregunta B15 y B17 (0.0001) se relaciona de modo coherente porque la lectura requiere de un espacio de un clima ambiental que contribuya a la concentración y al desarrollo de la creatividad para innovar y plantear problemas relacionados con la reorganización del conocimiento, al tiempo que toma decisiones para problematizar lo aprendido mediante la apropiación de habilidades de pensamiento de orden superior como la comparación, la generalización, la conclusión y la conceptualización, con el fin de lograr una autonomía intelectual, moral y científica.

\section{Medición del nivel de autogestión del aprendizaje}

Con el propósito de obtener una calificación global del nivel de autogestión del aprendizaje en los estudiantes de la población encuestada, se construyó la variable auxiliar CalfB con las respuestas de las 
preguntas B13 a B17 ponderadas según la tabla descrita en la tabla 6 y siguiendo la metodología descrita a continuación.

Se calcula el valor $\mathrm{m}=5$ que corresponde a la suma de las ponderaciones mínimas en cada pregunta.

Se calcula el valor $\mathrm{M}=25$ la suma de las ponderaciones de mayor valor en cada pregunta.

Para cada encuesta se calcula el valor Obs que corresponde a la suma de los valores de las ponderaciones de las respuestas observadas.

Finalmente se calcula el valor de la variable CalfA que proporciona una calificación estandarizada de la autogestión en la escala de 0 a 5 para cada encuesta, según la siguiente fórmula

$$
\text { Calf } B=5 * \frac{O b s-m}{M-m}
$$

Siguiendo la metodología descrita en la sección anterior, es decir, utilizando el coeficiente de determinación de un modelo de regresión múltiple de rango completo donde CalfB es la variable respuesta y las ponderaciones en cada encuesta de las respuestas a las preguntas B13 a B17 como variables explicativas, se observa que las preguntas más significativas según el número variables ponderadas incluidas en el modelo corresponden a la descripción dada en la tabla 7.

\section{Resultado}

Tabla 7. Número variables ponderadas incluidas en el modelo

\begin{tabular}{|c|r|r|}
\hline $\begin{array}{c}\text { NUMERO DE } \\
\text { VARIABLES }\end{array}$ & \multicolumn{1}{|c|}{ R CUADRADO } & \multicolumn{2}{c|}{$\begin{array}{c}\text { VARIABLES EN EL } \\
\text { MODELO }\end{array}$} \\
\hline 1 & 0.9664 & AV14 \\
\hline 1 & 0.96 & AV16 \\
\hline 2 & 0.9874 & AV14 AV17 \\
\hline 2 & 0.9864 & AV16 AV17 \\
\hline 3 & 0.9938 & AV13 AV15 AV17 \\
\hline 3 & 0.9932 & AV13 AV14 AV17 \\
\hline
\end{tabular}




\section{Comentario}

La variable AV14 se relaciona con la utilización de los datos, los hechos y los acontecimientos de las asignaturas, para formar conceptos con un sentido de utilidad en la vida cotidiana. Esta variable es muy significativa en la evaluación de la autogestión del aprendizaje con un porcentaje de explicación del 96.64\%. Entonces, los estudiantes tratan de conceptualizar y teorizar los contenidos de las disciplinas en un contexto de aprendizaje de hechos y acontecimiento que perciben en la vida cotidiana, con el propósito de declarar el conocimiento fáctico a través del lenguaje, que tiene un sentido de transferencia y aplicación en lo que el individuo percibe del entorno o medio que lo rodea, incorporando los conocimientos previos a la nueva información que modifica la estructura cognitiva.

Otra variable igualmente significativa es la variable AV16 (96\%) que se refiere a la actitud positiva para enfrentar el aprendizaje. Esto quiere decir que la autogestión del aprendizaje implica una actitud afectiva positiva o negativa que influye en el ropaje del pensamiento y en la conducta del individuo. Por tal razón, la afectividad tiene que ver con la autoestima y el grado de valoración cognitiva para enfrentar las circunstancias negativas de la vida y superar la adversidad y la frustración para postergar el logro de las metas propuestas.

En el conjunto de modelos que utilizan las posibles combinaciones de 2 variables explicativas, son las variables AV14, AV17 (98,74\%) que se relacionan con un conocimiento declarativo y un clima ambiental adecuado para favorecer la concentración y la resolución de problemas de aprendizaje. Lo anterior significa que el estudiante aprende transfiriendo conocimiento en contexto y desarrolla la creatividad cuando dispone del espacio y de los recursos físicos indicados para recuperar y procesar la información en situaciones diferentes de aprendizaje.

Igualmente, significativa es la combinación de las variables AV16, AV17 $(98,64 \%)$ que se refieren a un conocimiento actitudinal y las condiciones ambientales de estudio. Entonces, se aprecia que los estudiantes consideran la amistad como un puente importante para mantener las buenas relaciones interpersonales, que esta se constituye en un catalizador de aprendizaje colaborativo, de jalonamiento cognitivo para que 
el inexperto aprenda del más experto, de implementar técnicas procedimentales por iniciativa propia en torno a estrategias pedagógicas que faciliten la meta inteligencia, de mantener una actitud positiva como resultado de una interacción con los compañeros, de procesar la información para el desarrollo de las tareas de aprendizaje y de variables ambientales adecuadas en el crecimiento de la formación integral, tanto del individuo como del colectivo.

Las variables AV13, AV16 (97,53\%) retoman el acceso a la sociedad del conocimiento (habilidad para inferir conocimiento en Internet) mediante una actitud positiva de indagación y de exploración al alrededor de la formación del espíritu científico (curiosidad por descubrir y resolver problemas). Los medios tecnológicos, en especial el Internet, son muy atractivos para el estudiante y captan la motivación para navegar con relación a los conflictos cognitivos que parten de las actividades de aprendizaje con las personas, las tareas y el uso de procedimientos o técnicas. En cuanto mayor afectividad e interés se sienta por las bondades del Internet, mayores son las posibilidades de profundizar en los aprendizajes en la sociedad del conocimiento.

En los modelos con 3 variables explicativas, es la combinación de las variables AV13, AV15, AV17 (99,38\%) que los estudiantes navegan en Internet para acceder a fuentes de información relacionadas con sentencias y autores clásicos de la filosofía. Además, cuando se navega se toman decisiones con respecto a los tópicos leídos de las disciplinas que forman parte del plan de estudio y se requiere contar con muy buenas condiciones ambientales de estudio que posibiliten la concentración y la atención en el estudio.

Igualmente, significativa es la combinación de las variables AV13, AV14, AV17 (99,32\%) que comprenden la consulta en Internet como un escenario para aprender los conocimientos que se declaran con el lenguaje, los hechos, los acontecimientos de los contenidos disciplinares del mundo digital, lo que sucede dentro y fuera del aula de clase para lograr un aprendizaje significativo. Estos procesos involucran contar con un buen clima ambiental interior y social que favorezca la autogestión para descodificación y codificación de conceptos.

A ese mismo nivel de porcentaje se encuentra la combinación de las variables AV13, AV15, AV16 (99,32\%), las cuales están relacionadas 
con la consulta de Internet para abordar los problemas académicos, la lectura de escritos que permite la toma de decisiones y poseer una actitud positiva para enfrentar el aprendizaje. Esto significa que la autogestión del aprendizaje parte de la problematización del conocimiento por Internet, al tiempo que potencia el desarrollo de habilidades de pensamiento de orden superior producto de la lectura, siempre y cuando, los aprendices tengan una actitud positiva para enfrentar el fracaso y la derrota en el estudio.

\section{Análisis descriptivo de la variable CalfB}

Tabla 8. Estadísticas descriptivas de la variable CalfB

\begin{tabular}{|l|l|}
\hline \multicolumn{2}{|l|}{ Resultado } \\
\hline Media & 4.08828671 \\
\hline Error típico & 0.04628027 \\
\hline Mediana & 4.25 \\
\hline Moda & 4.5 \\
\hline Desviación estándar & 0.78267033 \\
\hline Varianza de la muestra & 0.61257284 \\
\hline Curtosis & 0.41489146 \\
\hline Coeficiente de asimetría & -1.03467191 \\
\hline Rango & 3.5 \\
\hline Mínimo & 1.5 \\
\hline Máximo & 5 \\
\hline Suma & 1169.25 \\
\hline Cuenta & 286 \\
\hline
\end{tabular}

Tabla 9. Histograma de frecuencias de los valores de la variable CALFB

\begin{tabular}{|l|l|l|l|l|}
\hline \multicolumn{5}{c}{ Resultado } \\
\hline \multicolumn{1}{|c|}{ CALFB } & \multicolumn{1}{c|}{ Frec. } & \multicolumn{1}{r|}{ Frec Acum } & Porce & \% Acum \\
\hline $1.95 *$ & 1 & 1 & 0.35 & 0.35 \\
\hline $2.25 * * * *$ & 7 & 8 & 2.45 & 2.8 \\
\hline $2.55 * * *$ & 6 & 14 & 2.1 & 4.9 \\
\hline
\end{tabular}




\begin{tabular}{|c|c|c|c|c|}
\hline \multicolumn{5}{|c|}{ Resultado } \\
\hline CALFB & Frec. & Frec Acum & Porce & $\%$ Acum \\
\hline $2.85 * * *$ & 5 & 19 & 1.75 & 6.64 \\
\hline $3.15 * * * * * * * * *$ & 20 & 39 & 6.99 & 13.64 \\
\hline $3.45 * * * * *$ & 12 & 51 & 4.2 & 17.83 \\
\hline $3.75 * * * * * * * * *$ & 19 & 70 & 6.64 & 24.48 \\
\hline 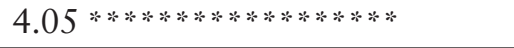 & 36 & 106 & 12.59 & 37.06 \\
\hline 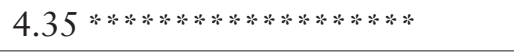 & 38 & 144 & 13.29 & 50.35 \\
\hline 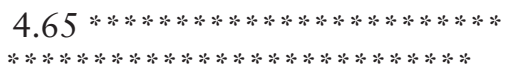 & 101 & 245 & 35.31 & 85.66 \\
\hline $4.95 * * * * * * * * * * * * * * * * * * * *$ & 41 & 286 & 14.34 & 100 \\
\hline
\end{tabular}

\section{Comentario}

El histograma de frecuencias de la variable CalfB muestra de manera global el nivel de autogestión del aprendizaje en los estudiantes encuestados. Como en la evaluación del nivel de autonomía para estandarizar la evaluación, el nivel de autogestión se reprueba si su nota es inferior a 3, se aprueba con un nivel de rendimiento aceptable si es superior o igual a 3 y menor de 3.7 y se aprueba con un nivel de rendimiento bueno si es superior a 3.7. Entonces, se observa que el $6.64 \%$ de los estudiantes tienen un nivel de autonomía inferior, el $11.18 \%$ tiene un nivel de autonomía aceptable y la gran mayoría, el $82.16 \%$ tiene un buen nivel de autonomía.

La tendencia de la calificación indica que la autogestión del aprendizaje para el desarrollo de la pedagogía del aprendizaje autónomo es decisiva porque los estudiantes establecen metas, organizan la información y acceden al Internet para ampliar y complementar los conocimientos, trabajan con un conocimiento declarativo y procedimental, descodifican y codifican conceptos y cuentan con un clima ambiental apropiado para aprender a aprender; aunque, un grupo representativo de estudiantes consideran que el Internet y los aparatos tecnológicos distraen y generan ruido que incide en el estudio. 


\section{Perfil del nivel de autogestión del aprendizaje dado el género}

Tabla 10. Perfil del nivel de autogestión del aprendizaje dado el género

\begin{tabular}{|l|c|c|c|}
\hline \multirow{2}{*}{\multicolumn{1}{|c}{ Género }} & \multicolumn{3}{|c|}{ Resultado } \\
\cline { 2 - 4 } & Aceptable & Bueno & Inferior \\
\hline Femenino & 0.12269939 & 0.80368098 & 0.07361963 \\
\hline Masculino & 0.09756098 & 0.84552846 & 0.05691057 \\
\hline
\end{tabular}

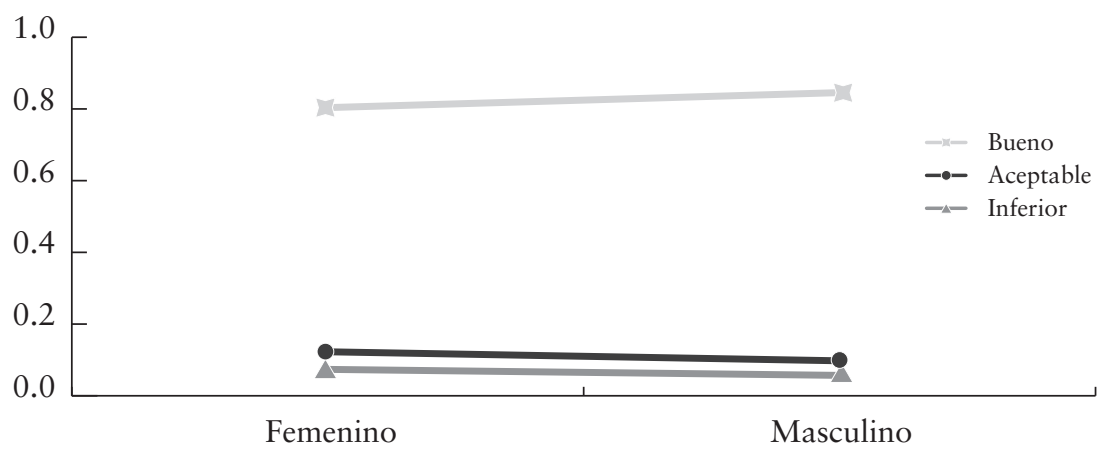

Figura 1. Perfil del nivel de autogestión del aprendizaje dado el género.

El nivel de autogestión del aprendizaje no depende del género en cuanto que el nivel de aceptable en el género femenino y masculino es similar. Este mismo fenómeno se repite en el nivel bueno y en el nivel inferior. Por tanto, el género no incide en el desarrollo de la autogestión del aprendizaje. 


\section{Perfil del nivel de autogestión del aprendizaje dado el número de semestres cursados}

Tabla 11. Perfil del nivel de autogestión del aprendizaje dado el número de semestres cursados

\begin{tabular}{|c|c|c|c|}
\hline \multirow{2}{*}{$\begin{array}{l}\text { Número de } \\
\text { semestres }\end{array}$} & \multicolumn{3}{|c|}{ Resultado } \\
\hline & Aceptable & Bueno & Inferior \\
\hline $\begin{array}{l}\text { Primeros } \\
\text { semestres }\end{array}$ & 0.10869565 & 0.85869565 & 0.0326087 \\
\hline $\begin{array}{l}\text { Semestres } \\
\text { intermedios }\end{array}$ & 0.11235955 & 0.78651685 & 0.1011236 \\
\hline Últimos semestres & 0.11428571 & 0.81904762 & 0.066666667 \\
\hline
\end{tabular}

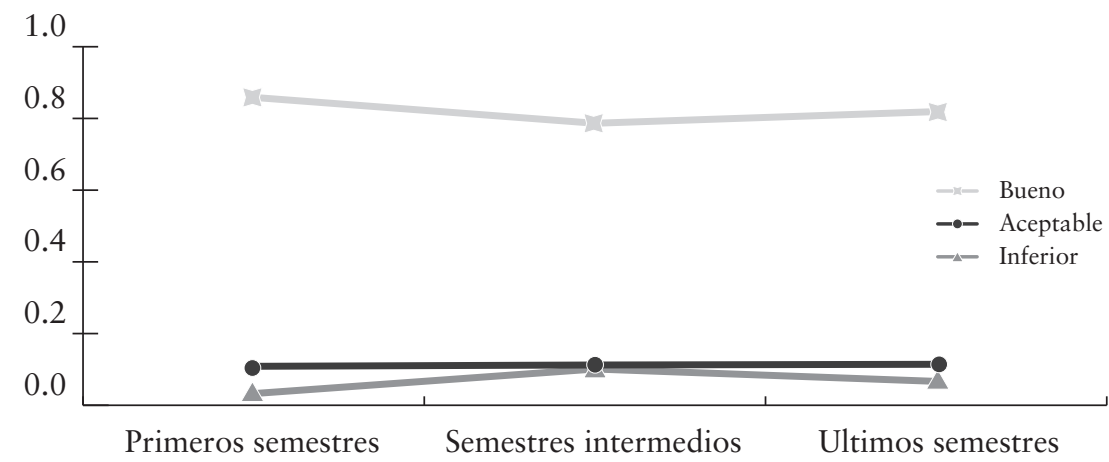

Figura 2. Perfil del nivel de autogestión del aprendizaje dado el número de semestres cursados.

La autogestión del aprendizaje no depende de los semestres cursados en cuanto que los niveles aceptable, bueno e inferior es muy similar en los primeros semestres, los semestres intermedios y los últimos semestres. Sin embargo, se aprecia que el semestre intermedio del nivel inferior presenta un leve incremento en torno a la autogestión del aprendizaje, esto puede obedecer a la necesidad de utilizar un repertorio de estrategias que lo conviertan en estudiante exitoso. Esto quiere decir que los estudiantes establecen acciones o procedimientos por iniciativa propia para llegar a una autonomía intelectual, moral y científica que conlleva a la transferencia y aplicación de conceptos dirigidos hacia 
la vida cotidiana, al tiempo que mejoran en el dominio de las competencias comunicativas (lectura y escritura) y disponen de un clima ambiental adecuado para estudiar.

\section{Perfil del nivel de autonomía dado el número promedio de horas fuera de clase para labores académicas}

Tabla 12. Perfil del nivel de autogestión dado el número promedio de horas fuera de clase para labores académicas

\begin{tabular}{|l|r|r|r|}
\hline \multirow{2}{*}{ Horas fuera de clase } & \multicolumn{3}{|c|}{ Resultado } \\
\cline { 2 - 4 } & \multicolumn{1}{|c|}{ Aceptable } & \multicolumn{1}{c|}{ Bueno } & \multicolumn{1}{l|}{ Inferior } \\
\hline Entre 11 y 15 & 0.07042254 & 0.83098592 & 0.09859155 \\
\hline Entre 5 y 10 & 0.112 & 0.824 & 0.064 \\
\hline Más de 16 & 0.07936508 & 0.88888889 & 0.03174603 \\
\hline Menos de 5 & 0.2962963 & 0.62962963 & 0.07407407 \\
\hline
\end{tabular}

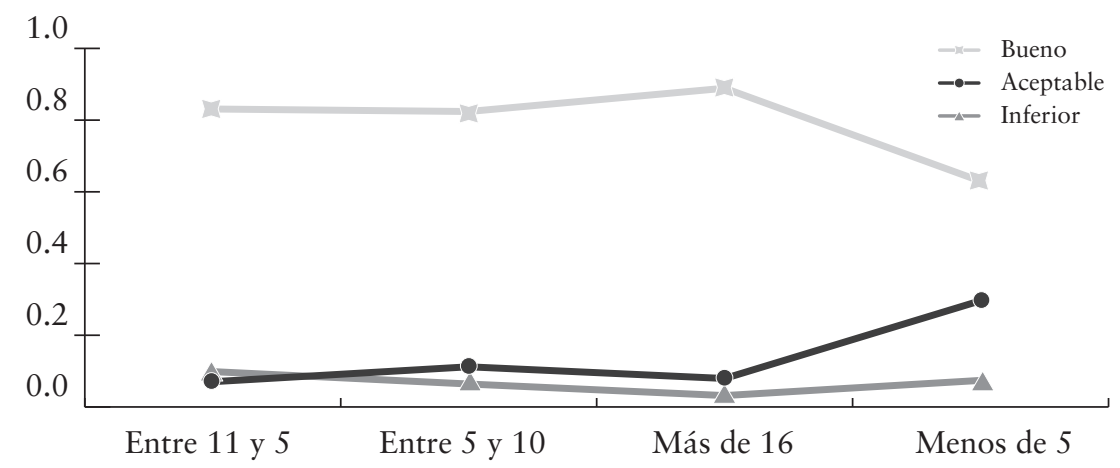

Figura 3. Perfil del nivel de autogestión del aprendizaje dado el número de horas fuera de clase para labores académicas.

Se aprecia que la mayoría de los estudiantes le dedican un nivel bueno de promedio de horas entre 11 y 15 y más de 16 horas fuera de clase para labores académicas. Sin embargo, se aprecia que otro grupo no le dedica el tiempo necesario al estudio. Por ende, se percibe que los 
estudiantes autogestionan el aprendizaje de acuerdo con el ritmo que se impongan de modo autónomo para acercarse a la información, fijar planes de estudio y autorregular los aprendizajes. Por tal motivo, la autogestión del aprendizaje depende de la motivación, el interés y el empeño para cumplir la meta propuesta en el proyecto de vida personal.

\section{Conclusiones}

En términos generales se plantea las conclusiones más relevantes, de acuerdo con los objetivos propuestos en el estudio.

- El Internet se constituye en una valiosa herramienta de indagación y de investigación para aprender sobre sentencias de índole nacional e internacional en el campo del derecho y de conocimiento de autores clásicos en torno a la filosofía. No obstante, los aparatos tecnológicos generan distracción y ruido, conllevando a la pérdida de la concentración y atención en el aula de clase que incide de forma indirecta en el rendimiento académico y en la autogestión del aprendizaje. Por otra parte, el Internet posibilita navegar para aprender sobre cuestiones de la vida y los problemas propios de las asignaturas del plan de estudio de las respectivas carrereas.

- La calificación de la utilidad de los conocimientos declarativos basados en hechos, acontecimientos, sucesos e ideas es decisiva porque facilita la trasferencia de conceptos y teorías en el ámbito de la praxis cotidiana de la vida, articulando de esta manera la teoría y la práctica con un sentido referido al aprendizaje significativo, sirviendo de anclaje las experiencias previas de la vida cotidiana para comprender los conceptos de las disciplinas. La iniciativa para abordar un conocimiento declarativo parte de los mismos estudiantes de Derecho y de la licenciatura en Filosofía y Lengua Castellana porque perciben como mediación la realidad social para entender la lógica y la dinámica de las teorías que forman parte de la problemática del hombre. 
- El impacto de la lectura permite al estudiante tomar decisiones entre varias teorías, propiciando la clarificación de la información, la generalización y la formulación de conclusiones. La lectura se presenta como un componente esencial para el desarrollo de habilidades de pensamiento de orden superior como la comparación, el análisis y la interpretación, siendo un catalizador determinante para tomar decisiones entre diversas teorías y conceptos en la solución de problemas del mundo de la vida del hombre. Del mismo modo, se percibe que el pensamiento hipotético no se explora como un ingrediente esencial en la problematización del conocimiento y que en última instancia potencia el desarrollo de la creatividad y la imaginación.

- Los estudiantes tienen una actitud positiva para enfrentar las circunstancias negativas de la vida. Esto obedece al compromiso y la autoobligación de formarse a sí mismos por la inversión económica que realiza la familia para proyectarse como futuro profesional con calidad. De igual manera, la actitud positiva asume la frustración y posterga el éxito, contribuyendo a la maduración de la inteligencia emocional, tan importante a futuro en la vida laboral y en la búsqueda de la felicidad en todo el sentido de la palabra, específicamente lo que tiene que ver con la motivación intrínseca para cumplir las metas personales.

- Las condiciones ambientales internas y sociales son claves para la autogestión del aprendizaje. Esto significa disponer de un espacio y de silencio, de comodidades físicas como un escritorio y una biblioteca para estudiar o de silencio, de un escritorio y un computador. Entonces, el ambiente de estudio es necesario para la concentración, el desarrollo de la creatividad para la realización de las tareas, de los ejercicios y de los compromisos adquiridos en el estudio. El clima ambiental es un factor determinante para el uso y generación de conocimiento, también un paso obligatorio para la adquisición de habilidades de pensamiento de orden superior que sirve de puente en la gestación de un pensamiento hipotético, tan necesario en la formación del espíritu científico. 
- La autogestión del aprendizaje se mide específicamente a partir de la variable del conocimiento declarativo y luego sigue la variable de actitud positiva. Esto significa que los estudiantes aprenden mediante hechos fácticos, lo cual facilita la conceptualización, el dominio de un conocimiento procedimental y actitudinal. Por ende, la realidad social actúa como un anclaje o polo a tierra para comprender los conceptos de las disciplinas con un ropaje de sentimientos y afectividad que incide en la conducta y el desarrollo de la creatividad con base en la nueva información adquirida, al tiempo que modifica la estructura cognitiva para que surjan nuevos conceptos y teorías.

\section{Referencias}

Aebli, H. (1998). Factores de la enseñanza que favorecen el aprendizaje autónomo. Madrid, España: Narcea.

Chaves, E. (2014). Autogestión del aprendizaje en la investigación educativa sobre Entornos personales de Aprendizaje (PLE): una revisión de la literatura Self - organized learning in Educacional research about Personal Learning Environmet (PLE): A review of literatura. Recuperado de http://www.edmetic.es/Documentos/Vol3Num2-2014/6.pdf

Fernández, R. (2005). Modelo informático para la autogestión del aprendizaje para la universalización de la enseñanza (Tesis Doctoral). Universidad de Granada. Recuperado de https://www.google.com.co/?gfe_rd=cr\&ei=lp4_ VbORHYuBsge694CwBQ\#q=tesis+doctoral+autogesti\%C3\%B3n+del+aprendizaje

Freire, P. (Eds.). (2009). Pedagogía de la autonomía. Saberes necesarios para la práctica educativa. México D.F., México: Siglo Veintiuno Editores.

Gardner, H. (1999). Estructuras de la mente. La teoría de las inteligencias múltiples. Santa de Bogotá. D. C., Colombia: Fondo de Cultura Económica.

Gaskins, I. y Elliot. T. (1999). Cómo enseñar estrategias cognitivas en la escuela. El manual Benchmark para docentes. Buenos Aires, Argentina: Paidós Educador.

Insuasty, L. D. (2001, 1 de agosto). El papel de la mediación en la autogestión del aprendizaje. Experiencias de mediación cognitiva, 1, 14-26. 
Insuasty, L. D. (2002, julio 3). La mediación didáctica: un sistema efectivo para promover el aprendizaje autónomo. Experiencias de mediación cognitiva. 3, 221-242.

Lorente. C, E. (2004). Autogestión en educación física. Un estudio de caso en secundaria (Tesis Doctoral). Universidad de Barcelona. Recuperado de http://www.tdx.cat/bitstream/handle/10803/2903/TESIS_ELORENTE.pdf? sequence $=1$

Lorente, E. y Joven, A. (2009). Autogestión en Educación Física: una investigación etnográfica. Recuperado de https://www.google.com. co/?gfe_rd=cr\&ei=7UVAVfn9EIHAgAS684GwCw\#q=la+tesis+doctoral+sobre+la+autogestion+del+aprendizaje\&start $=30$

Llinás, R. R. (2002). El cerebro y el mito del yo. Bogotá, Colombia: Editorial Norma.

Mateos, M. (2001). Metacognición y educación. Buenos Aires, Argentina: Aique.

Morin, E. (1999). La mente bien ordenada. Barcelona, España: Êditions du Seuil.

Ortega, A. (2007). Redin, modelo de creación de redes inteligentes de conocimiento para el fortalecimiento de la autogestión del aprendizaje. Recuperado de http://www.redalyc.org/pdf/761/76111485019.pdf

Pérez. L, A. (2010). Competencias para la autogestión del aprendizaje en las carreras de derecho y estudios socioculturales de la educación a distancia en la Universidad de Ciego de Ávila (Cuba) (Tesis Doctoral). Universidad de Granada. Recuperado de http://digibug.ugr.es/bitstream/10481/4970/1/18709217.pdf

Rué, J. (2009). El aprendizaje autónomo en la educación superior. Madrid, España: Narcea.

Varela, F. (2000). El fenómeno de la vida. Santiago de Chile, Chile: Editorial Dolmen.

Villardón, L. y Yániz, C. (2011). La autogestión del aprendizaje y la autonomía e iniciativa personal. Recuperado de http://dugi-doc.udg.edu/bitstream/handle/10256/3759/260.pdf? sequence $=1$ 



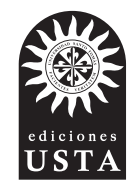

Esta obra se editó en Ediciones USTA, Departamento Editorial de la Universidad Santo Tomás. Se usó papel propalcote de 300 gramos para la carátula y papel bond beige de 75 gramos para páginas internas.

Tipografía de la familia Sabón.

Impreso por Image Printing, Ltda.

2017. 

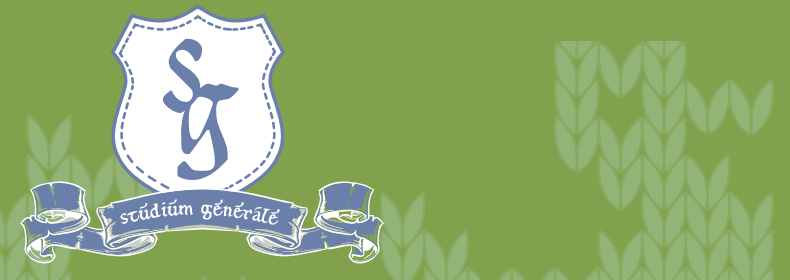

Punto de encuentro de las indagaciones de la Facultad de Filosofía y Letras de la Universidad Santo Tomás de Colombia, en este libro colectivo confluyen algunas de las búsquedas en filosofía, lenguaje y educación que persiguen los investigadores de esta escuela de humanidades. Por los caminos del filosofar, los lectores encontrarán acercamientos esclarecedores a la poesía en Platón, a la escritura en Benjamin, a la religión en Rosenzweig y Ricoeur. Por los caminos del pensamiento latinoamericano podrán rastrear las huellas metodológicas de la historia de las ideas, poéticas del sentipensar ontológico, decoloniales del pensamiento emergente en América Latina, políticas de la dialéctica entre poder y democracia. Por los caminos del lenguaje, seguirán los derroteros de la mística colonial de la madre Josefa del Castillo, de la novela del exilio de Santiago Gamboa, de la pragmática discursiva del fútbol. Y por los caminos pedagógicos, atravesarán las rutas del aprendizaje autónomo en la educación superior. En el cruce de caminos, se alza el esfuerzo compartido por pensar la cultura sobre y desde Colombia y América Latina.

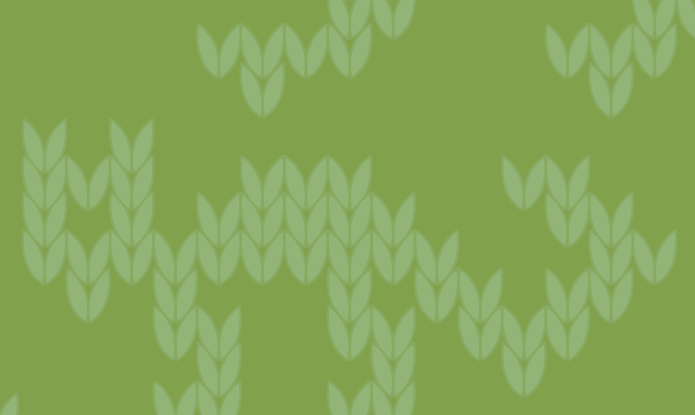

